\title{
Effect of Multiple Deconvolution Parameters on the Resolvability of Decay Rates of Multiexponential Signals
}

\author{
Abdussamad U. Jibia, Momoh-Jimoh E. Salami, and Othman O. Khalifa \\ Kulliyyah of Engineering \\ International Islamic University Malaysia \\ Jalan Gombak, 53100, Kuala Lumpur, Malaysia \\ E-mail: aujibia@gmail.com
}

Keywords: multiexponential, multiparameter deconvolution, eigenvector, pseudospectrum, Noise Reduction

\begin{abstract}
Noise reduction in deconvolution process has been a challenge to researchers in the field of signal processing. The problem is ill-posed and various algorithms have been developed to reduce noise enhancement. The effect of using multiple noise-compensating parameters in the deconvolution of multiexponential signals is considered in this paper. Three parameters are simultaneously adjusted to obtain optimal reduction in noise. It is shown that this approach performs better than a single parameter approach.
\end{abstract}

\section{INTRODUCTION}

Analysis of multiexponential signals with real decay rates proves to be difficult due to the nonorthogonality of the exponential function. This is more so when the signal is embedded in noise. There have been several attempts to analyse this class of signals (for example [1] and [4]-[6]) but the outcome has not always been without problems. One such attempt ([3], [7]) is to convert the original signal into a discrete convolution model whose input is a train of weighted delta functions containing the signal parameters to be determined. Fourier transformation and deconvolution then generate data consisting of a sum of complex exponentials in noise. The data is analyzed using spectral estimation techniques. This technique has proved to be efficient when used with SVD-based ARMA (Autoregressive Moving Average) [3], MUSIC (Multiple Signal Classification) and Minimum Norm Eigenvector [7] techniques. The main problem with this procedure is in the deconvolution stage. It is well known that deconvolution problems are generally ill-posed in the sense that the result of the deconvolution is highly sensitive to noise in the observed data and the system response function. A rational deconvolution procedure involves a trade-off between signal restoration and noise magnification. In [3], a single parameter was used in the deconvolution stage to minimize the effect of noise magnification. In this paper two more parameters have been introduced to further minimize this effect.

Though the concept of multiparameter deconvolution has been used in other applications, its introduction into multicomponent transient signal analysis is novel and it is the major contribution of this paper.

\section{THE CONVOLUTION MODEL}

Multicomponent signal can generally be expressed as

$$
S(\tau)=\sum_{k=1}^{M} A_{k} p\left(\lambda_{k} \tau\right)+n(\tau), \quad 0<\tau<\infty
$$

where $n(\tau)$ is noise, assumed to be white Gaussian.

In our own case, the basis function $p(\tau)=\exp (-\tau)$.

Equation (1) can be rewritten as

$$
S(\tau)=\int_{0}^{\infty} g(\lambda) p(\lambda \tau) d \lambda+n(\tau)
$$

where

$$
g(\lambda)=\sum_{k=1}^{M} A_{k} \delta\left(\lambda-\lambda_{k}\right)
$$

and it contains all the parameters to be determined.

Multiplying both sides of (3) by $\tau^{\alpha}$ and applying the Gardner transformation, $\tau=\mathrm{e}^{t}$ and $\lambda=\mathrm{e}^{-r}$ results in the convolution integral

$$
y(t)=\int_{-\infty}^{\infty} x(\lambda) h(t-\lambda) d \lambda+v(t)
$$

where

$$
\begin{aligned}
& y(t)=\exp (\alpha t) S\{\exp (t)\}, \\
& \left.x(t)=\exp \{(\alpha-1) t\} g\left(e^{-t}\right)\right\}, \\
& h(t)=\exp (\alpha t) p\left(e^{t}\right), \text { and } \\
& v(t)=\exp (\alpha t) n\left(e^{t}\right)
\end{aligned}
$$

Taking the Fourier transform of (4) and performing inverse filtering followed by inverse Fourier transformation yields

$$
x(t)=\sum_{k=1}^{M} B_{k} \delta\left(t+\ln \lambda_{k}\right)
$$

where $B_{k}=A_{k}\left(\lambda_{k}\right)^{-\alpha}$.

A discrete form of (4) is obtained by sampling $y(t)$ at a rate of $1 / \Delta t \mathrm{~Hz}$, yielding the discrete convolution 


$$
y[n]=\sum_{m=-n_{\min }}^{n_{\max }} x[m] h[n-m]+v[n]
$$

Where $N=n_{\max }-n_{\min }+1, n_{\max }$ and $n_{\min }$ represent respectively the upper and lower data cut-off points.

\section{MULTIPARAMETER DECONVOLUTION}

Taking the DFT (Discrete Fourier Transform) of (6) yields

$$
Y(k)=X(k) H(k)+V(k)
$$

from which the deconvolved data can be generated according to

$$
\hat{X}(k)=\frac{Y(k)}{H(k)}=X(k)+\frac{V(k)}{H(k)},
$$

for $0 \leq k \leq N$-1, where $Y(k), X(k), H(k)$, and $V(k)$ represent respectively the DFT of $y(n), x(n), h(n)$, and $v(n)$.

The deconvolution process, as is well known, enhances noise. For low-frequency signals, which is usually the case, deconvolution favors high frequency components in the data and hence the noise embedded in the signal. Worse still, even if the original data were noise free, the operation will result in some noise due to computational errors and this noise is enhanced by the deconvolution process.

To reduce the effect of noise enhancement, an optimally compensated inverse filtering procedure was used in [3]. In that approach, a regularizing parameter $\mu$ was introduced in the deconvolution process and the deconvolved data is generated according to:

$$
\hat{X}(k)=\frac{Y(k) H^{*}(k)}{\left[|H(k)|^{2}+\mu\right]},
$$

where the symbol * denotes complex conjugate. As SNR is decreased, $\mu$ was adjusted until an optimal value is found that produces the best output.

To further enhance noise reduction, a second order difference operator model is introduced as follows:

By considering the output to be the DFT of the second order difference operator $\mathrm{B}(\mathrm{k})$, the transfer function will be:

$$
L(k)=\frac{B(k)}{X(k)}
$$

$\mathrm{B}(\mathrm{k})$ is derived as follows:

$$
\begin{aligned}
& b(n)=\nabla^{2} x(n)=\nabla x(n)-\nabla x(n-1) \\
& =x(n)-x(n-1)-(x(n-1)-x(n-2)) \\
& =x(n)-2 x(n-1)+x(n-2)
\end{aligned}
$$

Taking the z-transform of $b(n)$ yields:

$$
\begin{aligned}
& B(z)=X(z)\left(1-2 z^{-1}+z^{-2}\right) \\
& =X(z)\left(1-z^{-1}\right)^{2}
\end{aligned}
$$

The DFT follows

$$
\begin{aligned}
& B\left(e^{j \omega}\right)=X\left(e^{j \omega}\right)\left(1-e^{j \omega}\right)^{2} \\
& =X\left(e^{j \omega}\right)\left(e^{-j \omega / 2} \cdot 2 j\right)^{2}\left(\frac{e^{j \omega / 2}-e^{-j \omega / 2}}{2 j}\right)^{2}
\end{aligned}
$$

Thus

$L\left(e^{j \omega}\right)=\frac{B\left(e^{j \omega}\right)}{X\left(e^{j \omega}\right)}=-4 e^{-j \omega}\left(\frac{e^{j \omega / 2}-e^{-j \omega / 2}}{2 j}\right)^{2}$

Taking $\omega=\frac{2 \pi k}{N}$, we have

$$
L(k)=-4 e^{-j \frac{2 \pi k}{N}}\left(\sin ^{2}\left(\frac{\pi k}{N}\right)\right)
$$

Hence,

$$
|L(k)|^{2}=16 \cdot \sin ^{4}\left(\frac{\pi k}{N}\right)
$$

Equation (15) can be combined with (7) to give

$$
\hat{X}(k)=\frac{Y(k) H^{*}(k)}{|H(k)|^{2}+\mu+\alpha|L(k)|^{2}}
$$

where $\alpha$ determines the contribution of the additional term.

This same procedure can be used to derive any additional term of higher even order which can be used to generate high quality deconvolved data. Combining (16) with the fourth-order difference operator gives an inverse filter of the form

$$
\hat{X}(k)=\frac{Y(k) H^{*}(k)}{|H(k)|^{2}+\mu+\alpha|L(k)|^{2}+\beta|L(k)|^{4}}
$$

Thus, the three regularizing parameters are $\alpha, \beta$ and $\mu$ which can be optimized to compensate for the noise.

\section{EIGENVECTOR SIGNAL PROCESSING}

Denoting the truncated deconvolved data as $f(k)$ and based on (5) and any of (7), (8) and (17), we have

$$
f(k)=\sum_{i=1}^{M} A_{i} \exp \left\{j k \Delta \omega \ln \lambda_{i}\right\}+\varepsilon(k)
$$

for $k=1,2, \ldots \ldots, 2 N_{0}+1 ; N_{0} \leq(N / 2)-1$ is the truncation point and $\varepsilon(k)$ is the deconvolved noise. 
The MUSIC Algorithm for this type of signal was developed in [7] as:

$$
\bar{R}_{\text {musi }}\left(e^{j \Delta \omega \ln \lambda)}=\frac{1}{\left.\sum_{p=M+1}^{P} r^{H}(\ln \lambda) v_{p}\right|^{2}}=\frac{1}{\sum_{p=M+1}^{P}\left|V_{p}\left(e^{j \Delta \omega \ln \lambda}\right)\right|^{2}}\right.
$$

An assumption inherent in the MUSIC pseudospectrum is that noise eigenvalues all have equal powers, that is, the noise is white. In practice however, the noise eigenvalues will not be equal. The differences become more pronounced when the correlation matrix is estimated from a small number of data samples. Thus a slight modification in the MUSIC algorithm was proposed [2] to account for the potentially different noise eigenvalues. The modification when incorporated into (12) yields:

$$
\bar{R}_{\text {mon } u \leq x}=\frac{1}{\sum_{M+1}^{p} \frac{1}{\lambda_{y}}\left|r^{H}(\ln \lambda) v_{y}\right|^{2}}=\frac{1}{\sum_{M+1}^{p} \frac{1}{\lambda_{y}}\left|V_{p}\left(e^{j \operatorname{lah} \lambda}\right)\right|^{2}}
$$

where $\lambda_{p}$ is the eigenvalue associated with the eigenvector $\mathrm{v}_{\mathrm{p}}$. The pseudospectrum of each eigenvector is normalized by its corresponding eigenvalue. In the case of equal noise eigenvalues $\left(\lambda_{\mathrm{p}}=\sigma_{\mathrm{w}}{ }^{2}\right)$ for $\mathrm{M}+1 \leq \mathrm{p} \leq \mathrm{P}$, the two pseudospectrums are the same.

\section{SIMULATION RESULTS}

In order to make an easy comparison, two signals used in [3] and [7] were used. In both cases only one parameter, $\mu$ was used in the deconvolution stage and performance was found to deteriorate below the threshold SNR (Signal to Noise Ratio) of $43 \mathrm{~dB}$ in the case of [3] and $40 \mathrm{~dB}$ in the case of [7]. However, when two more regularizing parameters, $\alpha$ and $\beta$ were used good performance was recorded up to about $\mathrm{SNR}$ of $35 \mathrm{~dB}$ for five components and $30 \mathrm{~dB}$ for two components.

The two signals are:

$$
\begin{aligned}
& S_{1}(\tau)=0.5 e^{-0.5 \tau}+e^{-\tau}+2 e^{-2 \tau}+5 e^{-5 \tau}+10 e^{-10 \tau}+n(\tau) \\
& S_{2}(\tau)=0.5 e^{-0.5 \tau}+e^{-\tau}+n(\tau)
\end{aligned}
$$

Fig. 1 through fig. 4 show selected pseudospectral plots for $\mathrm{S}_{1}(\tau)$ and $\mathrm{S}_{2}(\tau)$ for two different sets of parameters (i.e. SNR, $\alpha, \beta$ and $\mu)$ each. The estimated parameters $(\ln \lambda)$ are given in Tables 1 and 2 for the same conditions.

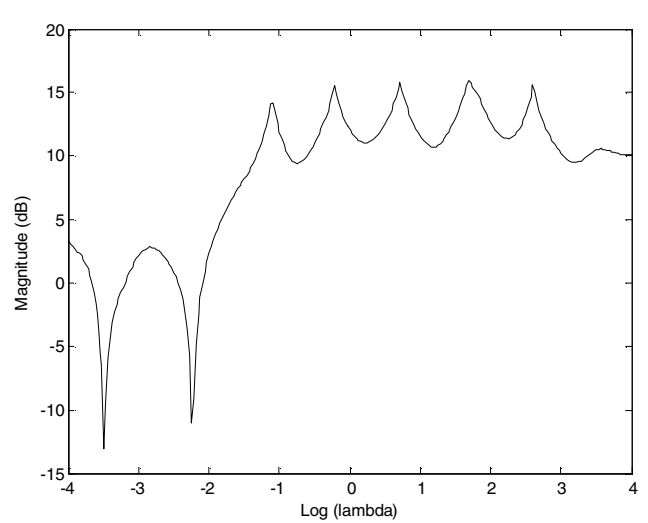

Fig. 1. Pseudospectrum for $S_{1}(\tau)$ with $S N R=31 d B, \mu=\alpha=-300 d B, \quad \beta=-$ $500 \mathrm{~dB}$

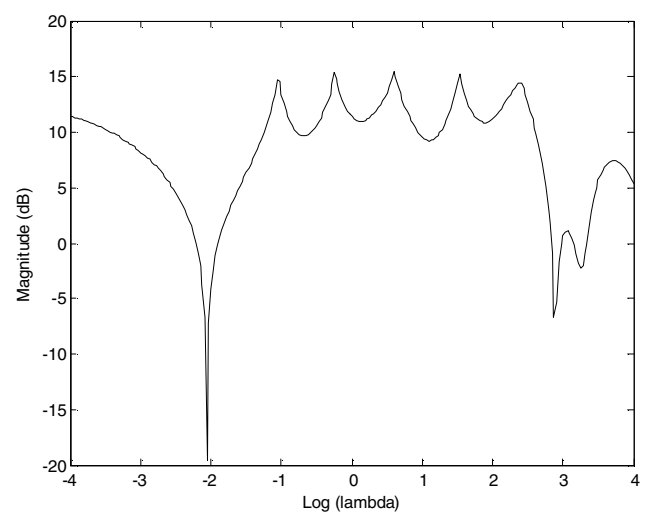

Fig. 2. Pseudospectrum for $\mathrm{S}_{1}(\tau)$ with $\mathrm{SNR}=34.8 \mathrm{db}, \mu=-200 \mathrm{~dB}$, $\beta=-300 \mathrm{~dB}, \alpha=-250 \mathrm{~dB}$

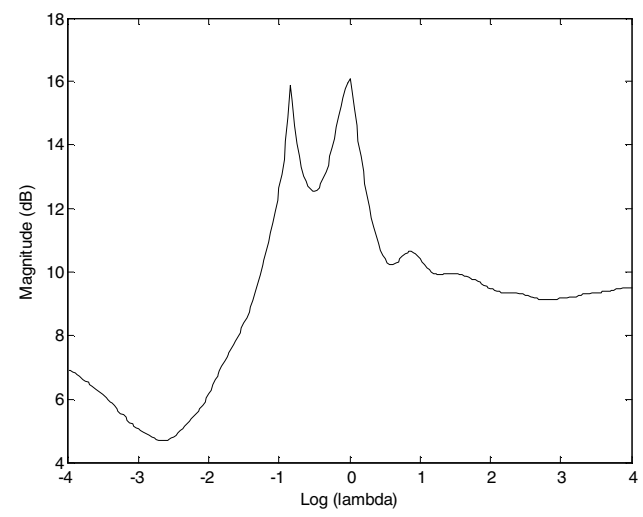

Fig. 3. Pseudospectrum for $\mathrm{S}_{2}(\tau)$ with $\mathrm{snr}=30.5 \mathrm{~dB}, \mu=-130 \mathrm{~dB}$, $\alpha=-200 \mathrm{~dB}, \beta=-500 \mathrm{~dB}$ 


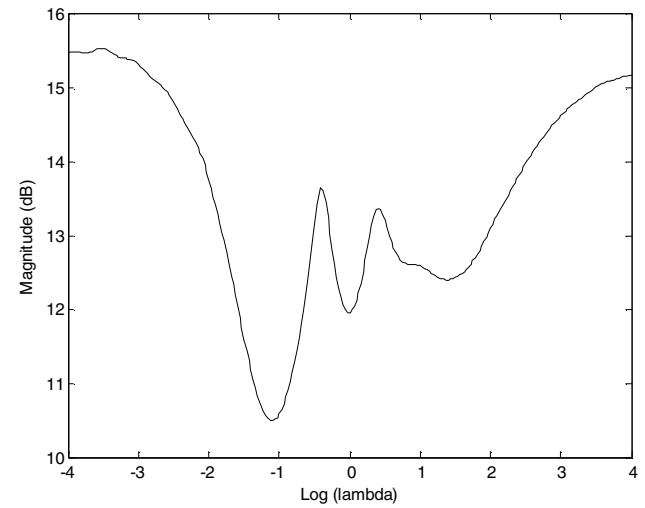

Fig. 4. Pseudospectrum for $\mathrm{S}_{2}(\tau)$ with $\mathrm{SNR}=25 \mathrm{~dB}, \mu=-130 \mathrm{~dB}$, $\beta=-500 \mathrm{~dB}, \alpha=-200 \mathrm{~dB}$

\begin{tabular}{||c|l|l|l|l|l|l||}
\hline \hline conditions & $\ln \lambda_{1}$ & $\operatorname{Ln} \lambda_{2}$ & $\operatorname{Ln} \lambda_{3}$ & $\operatorname{Ln} \lambda_{4}$ & $\operatorname{Ln} \lambda_{5}$ & Remarks \\
\hline $\begin{array}{c}\mathrm{SNR}=31 \mathrm{~dB} \\
\beta=-500 \mathrm{~dB} \\
\mu=\alpha=-300 \mathrm{~dB}\end{array}$ & -1.094 & -0.2188 & 0.7188 & 1.688 & 2.594 & $\mathrm{Bad}$ \\
\hline $\begin{array}{c}\mathrm{SNR}=34.80 \mathrm{~dB} \\
\beta=-300 \mathrm{~dB} \\
\mu=-200 \mathrm{~dB} \\
\alpha=-250 \mathrm{~dB}\end{array}$ & -0.875 & -0.0625 & 0.75 & 1.625 & 2.469 & Good \\
\hline $\begin{array}{c}\text { Expected } \\
\text { Value }\end{array}$ & -0.6932 & 0 & 0.6932 & 1.6094 & 2.3026 & \\
\hline
\end{tabular}

Table 1. Values of $\ln \lambda$ For 2 Different Conditions For $\mathrm{S}_{1}(\tau)$

\begin{tabular}{|c|l|l|l||}
\hline conditions & $\ln \lambda_{1}$ & $\ln \lambda_{2}$ & Remarks \\
\hline $\mathrm{SNR}=25 \mathrm{~dB}$ & -0.4063 & 0.4063 & Bad \\
$\beta=500 \mathrm{~dB}$ & & & \\
$\mu=-130 \mathrm{~dB}$ & & & \\
$\alpha=-200 \mathrm{~dB}$ & & & \\
\hline $\mathrm{SNR}=30.5 \mathrm{~dB}$ & -0.8348 & 0.4063 & Good \\
$\beta=500 \mathrm{~dB}$ & & & \\
$\mu=-130 \mathrm{~dB}$ & & & \\
$\alpha=-200 \mathrm{~dB}$ & & & \\
\hline Expected Value & 0.69315 & 0 & \\
\hline \hline
\end{tabular}

. Table 2. Values of $\ln \lambda$ for Different Conditions For $\mathrm{S}_{2}(\tau)$

\section{CONCLUSION}

In this paper, a three-parameter deconvolution model for the analysis of multiexponential signals with real decay rates has been developed and tested. By suitably adjusting the parameters better performance was recorded at lower SNR than in the case of a single regularizing parameter.

\section{REFERENCES}

[1] D.G. Gardner, J.C. Gardner, G. Lush, and W.R. Ware, "Method for the analysis of multicomponent exponential decay curves", Journal of Chemical Physics, vol. 31, pp. 978-986, 1959.

[2] M..H. Hayes, Statistical Signal Processing and Modeling, John Wiley and sons, Inc., New York, 1996.

[3] M.J.E. Salami, and S.N. Sidek, "Parameter estimation of multicomponent transient signals using deconvolution and ARMA modeling techniques", Journal of Mechanical systems and signal processing, Academic Press, UK, Vol. 17, Issue 6, pp. $1201-1218,2003$.

[4] D. F. Eaton, "Recommended methods for fluorescence decay analysis", Journal of Pure and Applied Chemistry, vol. 62, No 8, pp. 1631-1648, 1990.

[5] D.N. Swingler, "A differential technique for the Fourier transform processing of multicomponent exponential functions". IEEE transactions on biomedical engineering, BME-24(4): pp. 408-410, 1977.

[6] A. Jasbir, "Multiexponential Signal Analysis using the Total Least Squares Method", M.Sc. Dissertation, Dept of Electrical Engineering, University of Calgary, Canada, 1990.

[7] A. U. Jibia, \& M. J. E., Salami, "Performance Evaluation of MUSIC and Minimum Norm Eigenvector Algorithms in the Analysis of Noisy Multiexponential Signals", International Journal of Computer Science, Vol. 2, No. 4: pp. 235-239, 2007. 